\title{
Preliminarily Results from A Mini Neutron Monitor in Central Saudi Arabia
}

\author{
Maghrabi A. ${ }^{1 a}$,Al Mutairi $M^{a}$., Aldosary $A^{a}$, Altlasi $M^{a}{ }^{a}$, Alshehri $A^{a}$., Alfadhel \\ $\mathbf{R}^{\mathrm{b}}$., Almshari $\mathbf{B}^{\mathrm{b}}$., Alrashied $\mathbf{J}^{\mathrm{b}}$., Almutairi $\mathrm{E}^{\mathrm{b}}$., Alrashide $\mathrm{B}^{\mathrm{b}}$. \\ a National Centre for Applied Physics, King Abdulaziz City for Science and Technology, \\ 6086Riyadh 11442, Saudi Arabia. \\ b Prince Nora Bin Abdulaziz University, Riyadh 844428, Saudi Arabia.
}

Email:amaghrabi@kacst.edu.sa

\begin{abstract}
Different types of ground-based detectors have been developed and deployed around the world to monitor and study cosmic ray (CR) variations. As part of the King Abdulaziz City for Science and Technology (KACST) space research program and radiation detector laboratory activities, a mini neutron monitor was installed at (KACST) central Saudi Arabia (Riyadh; 24 43; long. 4640 ; alt. $613 \mathrm{~m}$; $\mathrm{Rc}=14.4 \mathrm{Gv}$ ) for continuous measurement of the cosmic ray neutrons and exploring their variations. In this paper, the technical aspects of this detector will be briefly discussed. Comparisons of the results obtained from this detector with those from the existing $1 \mathrm{~m}^{2}$ scintillator detector will be presented. The effects of atmospheric pressure on the collected data will be studied. Power spectral analyse were applied for the collected data to identify the short term periodicities.
\end{abstract}

36th International Cosmic Ray Conference -ICRC2019-

July 24th - August 1st, 2019

Madison, WI, U.S.A.

\footnotetext{
*M Al Mutairi

(C) Copyright owned by the author(s) under the terms of the Creative Commons 


\section{Introduction}

Extensive measurements of cosmic ray (CR) variations in space and on the ground began more than 90 years ago. These measurements have been conducted by diffrent instruments that were sensitive to various components of CRs and operated at different latitudes, including instruments carried by balloons [e.g., 1 and references therein]. Monitoring CR in Saudi Arabia began in 2002 when the first CR detector was installed. Since then, several CR detector have been developed and installed to study and explore the variations of CRs. For the same purposes, a mini-neutron monitor has been, recently, installed at King Abulaziz City for Science and Technology (KACST) central Saudi Arabia (Riyadh; Rc =14.4 Gv) to complement the existing detectors. This detector was built by the Centre of Space Research (North-West University, Potchefstroom, South Africa) [2-3]. In this paper, preliminarily results obtained from this detector will be presented.

\section{The Mini Neutron Monitor (NM)}

The technical details and calibration procedures of the detector have been discussed in several research articles [2-5]. The detector (F.g. 1) has a dimension about 1/3 of the original size of the typical full size NM64 neutron monitor, yet its actual count rate is same as the standard NM64 detector. A standard neutron monitor is designed as a counter surrounded with consecutive layers of a light-element material. For the mini NM, the outer paraffin wax reflector $(9.5 \mathrm{~cm})$ shields thermal nucleons and is transparent to cascade neutrons, which are multiplied in the lead producer. The inner, $2 \mathrm{~cm}$ thick, paraffin wax moderator was surrounded by a $5 \mathrm{~cm}$ neutron producer made of lead. Finally, multiplied and decelerated neutrons reach the $63 \mathrm{~cm}$ long LND2043 counter which was filled by BF3 gas with a pressure of $933 \mathrm{hPa}$. The detector was entirely controlled by a PIC32 microcontroller. An interface unit was developed for logging the data, interfacing with the electronics, and storing the data. The detector is located inside the radiation detector laboratory at KACST and has been in operation since April 2017. 


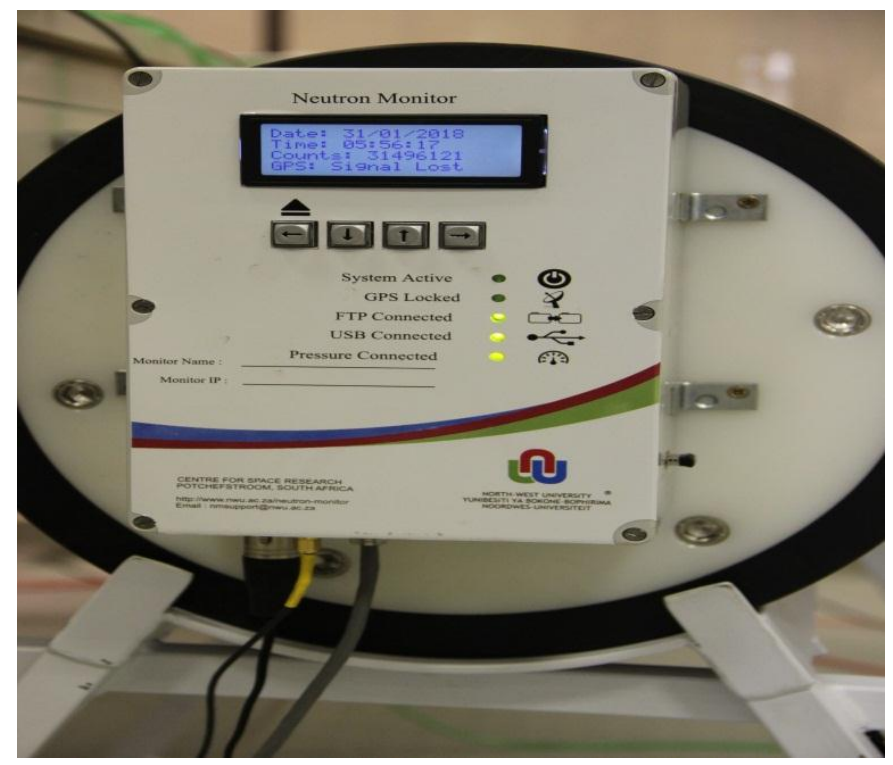

Fig. 1: photo shows the KACST mini Neutron Monitor (NM).

\section{Results and Discussions}

\subsection{Atmospheric pressure and CR Neutrons}

Atmospheric pressure determines the atmospheric mass that the $\mathrm{CR}$ particles need to go through before reaching the detector [e.g., 6].

The pressure effect on the secondary cosmic ray component can be expressed as:

$\frac{\mathrm{I}-\mathrm{I}_{0}}{\mathrm{I}_{0}}=\alpha\left(\mathrm{P}-\mathrm{P}_{0}\right)$

I is the cosmic ray rate at pressure $\mathrm{P}, \mathrm{I}_{0}$ is the rate at the mean atmospheric pressure at the site altitude (here $\mathrm{P}_{0}=940$ mbar), and $\alpha$ is the barometric coefficient.

Hourly values of the CR neutrons are plotted against the atmospheric pressure as indicated in Fig.2. Correlation analysis between the two variables gives a barometric coefficient of $0.65 \pm 0.01 \% / \mathrm{hPa}$ with a 0.65 correlation coefficient $(\mathrm{r})$. The obtained coefficient was used in this study to remove the effect of the atmospheric pressure from the collected data. 


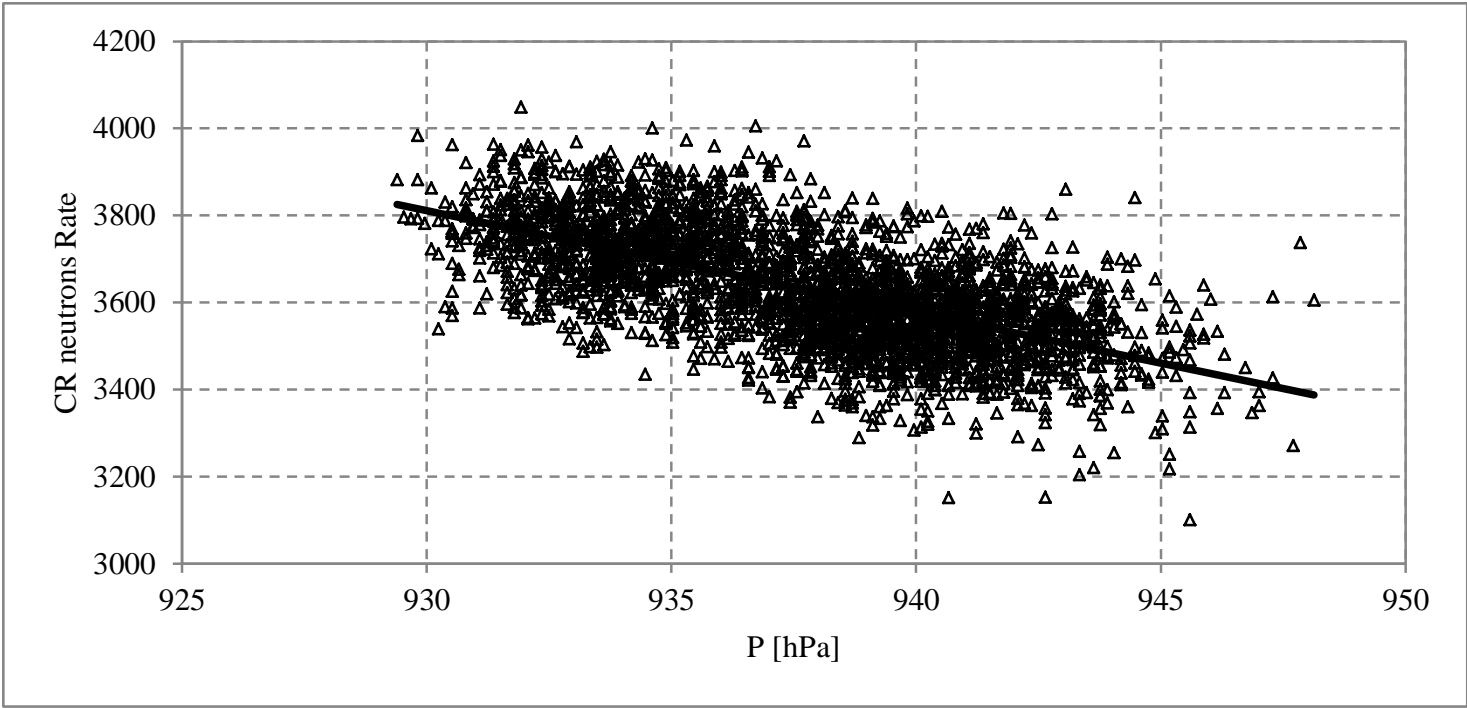

Fig. 2: Scatter plot between the hourly values of atmospheric pressure and CR neutrons during the study period. The straight line is the best linear fit.

In order to test the performance of the mini neutron monitor, cosmic ray data acquired from KACST muon detector ,installed at the same site, were utilized for this purpose. The KACST detector was discussed in several research papers [e.g., 7-8]. Pressure corrected CR data from both detectors for the period from 5 April to 20 April are depicted in fig.3. It is obvious that the behavior of the CRs observed by the mini NM are consistent with those detected by the muon detector for most of the period.

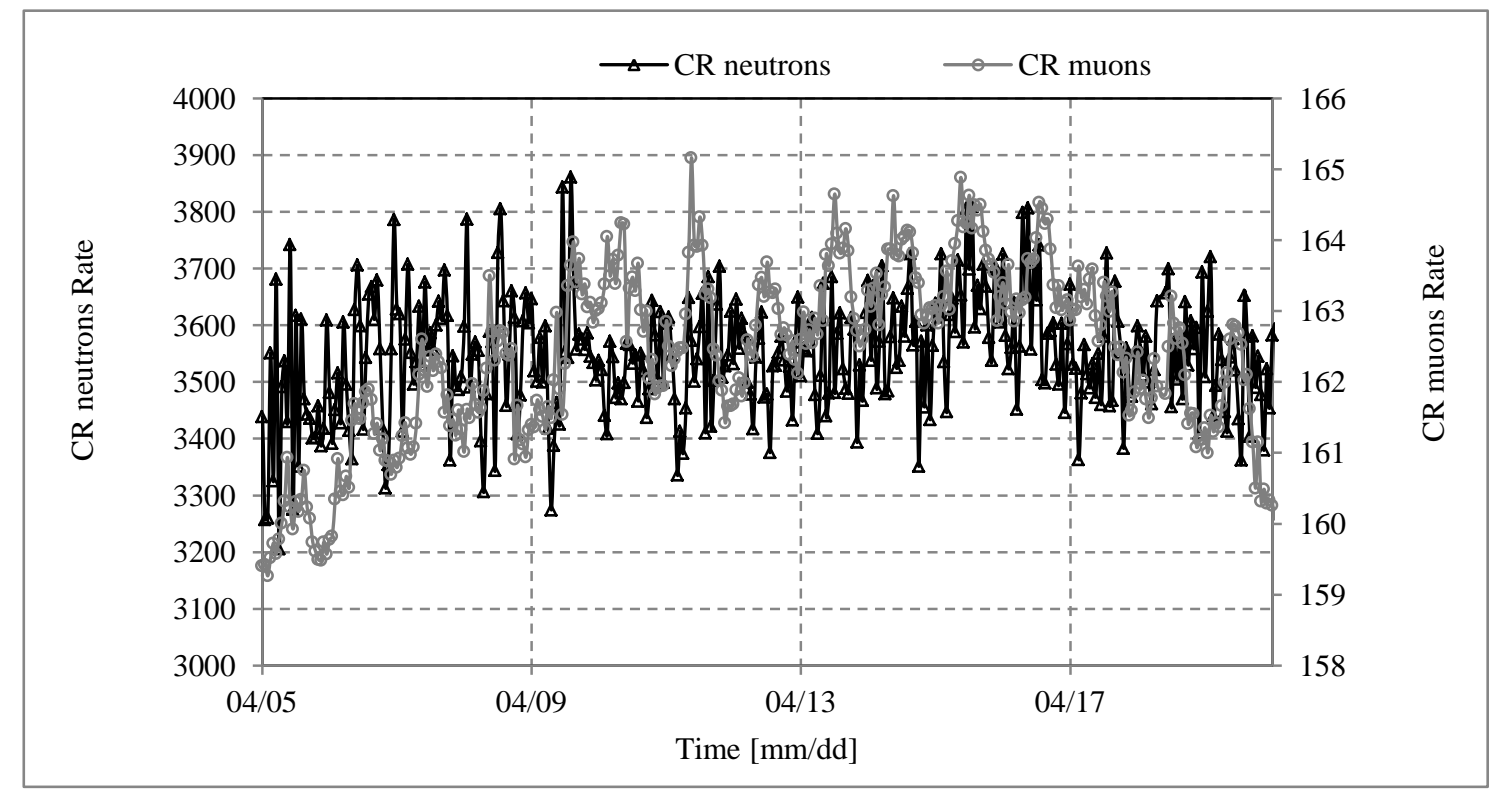

Fig. 3: Hourly variations of the CR muons recorded by KACST muon detector and CR neutrons recorded by the mini NM for the period between 5-20 April 2017. 


\subsection{CR Neutrons Diurnal Variation}

Daily variations of the CRs are due to the daily rotation of the earth around the sun, which results recording CRs with different intensities. Fig.4 shows the daily variations of the CR neutrons recorded by the mini NM during the study period. It is clearly seen that the maximum intensity occurred at 16:00 h LT corresponding to 13:00 h UT, whereas the minimum occurred at 05:00 LT. This result is consistent with those obtained previously by several researchers [e.g., 9-10].

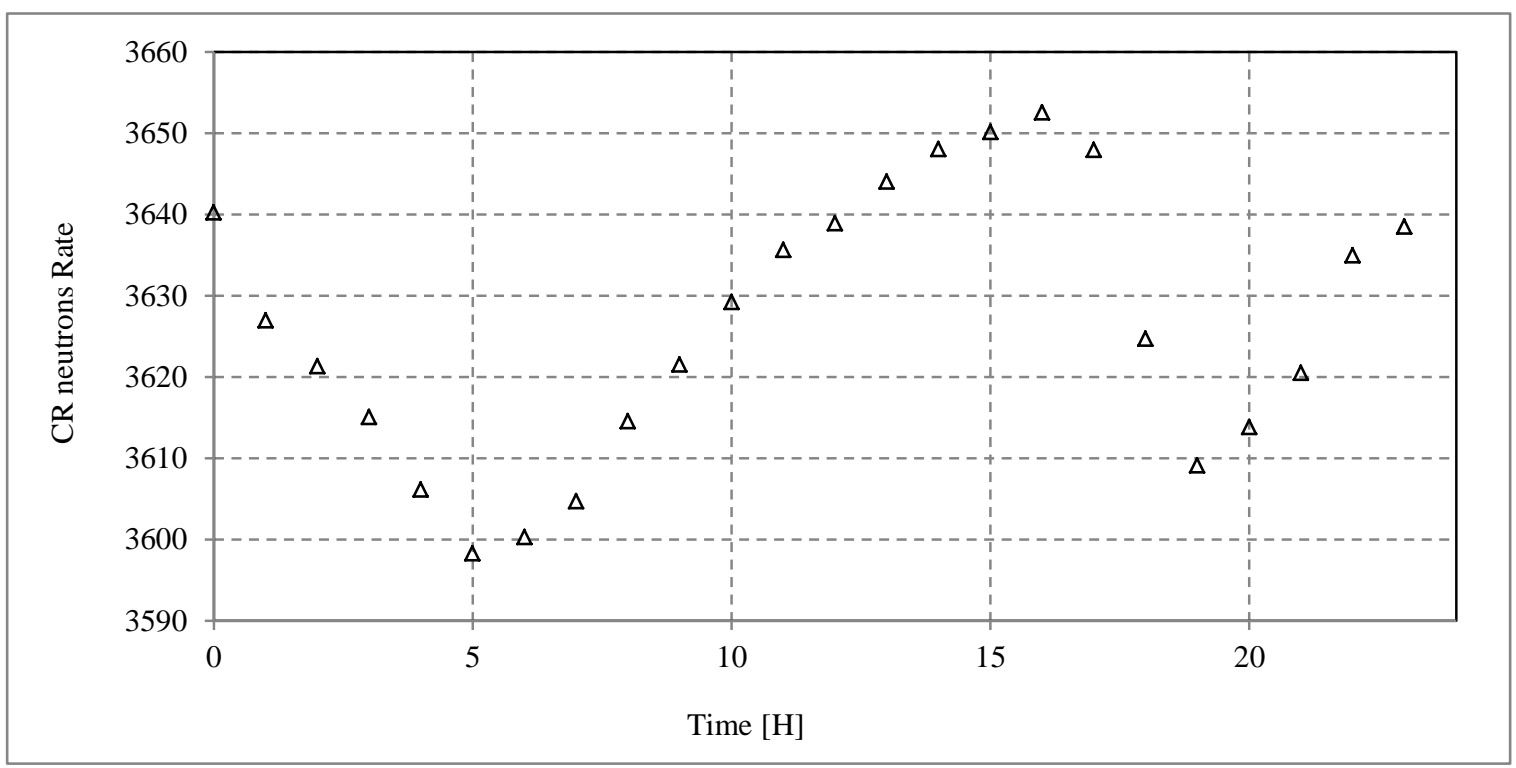

Fig. 4: Daily variation of the pressure corrected CR neutrons recorded by the mini NM during the study period.

\subsection{Cosmic Ray Neutrons Periodicities}

In this section power spectral analyses using Fourier Transform (FT) technique were carried out to investigate the periodicities in the CR neutrons collected by the mini NM. Fig. 5 is a power spectral density shows the main peaks of the CR neutrons. The neutron monitor has several significant peaks. These are the 36 days, 23 days, 12.5 days, 7.4 days, 5.6 days, 4.9 days, and 3.5 days. The obtained periodicities are in agreements with those found by several investigators [e.g., 11-13]. 


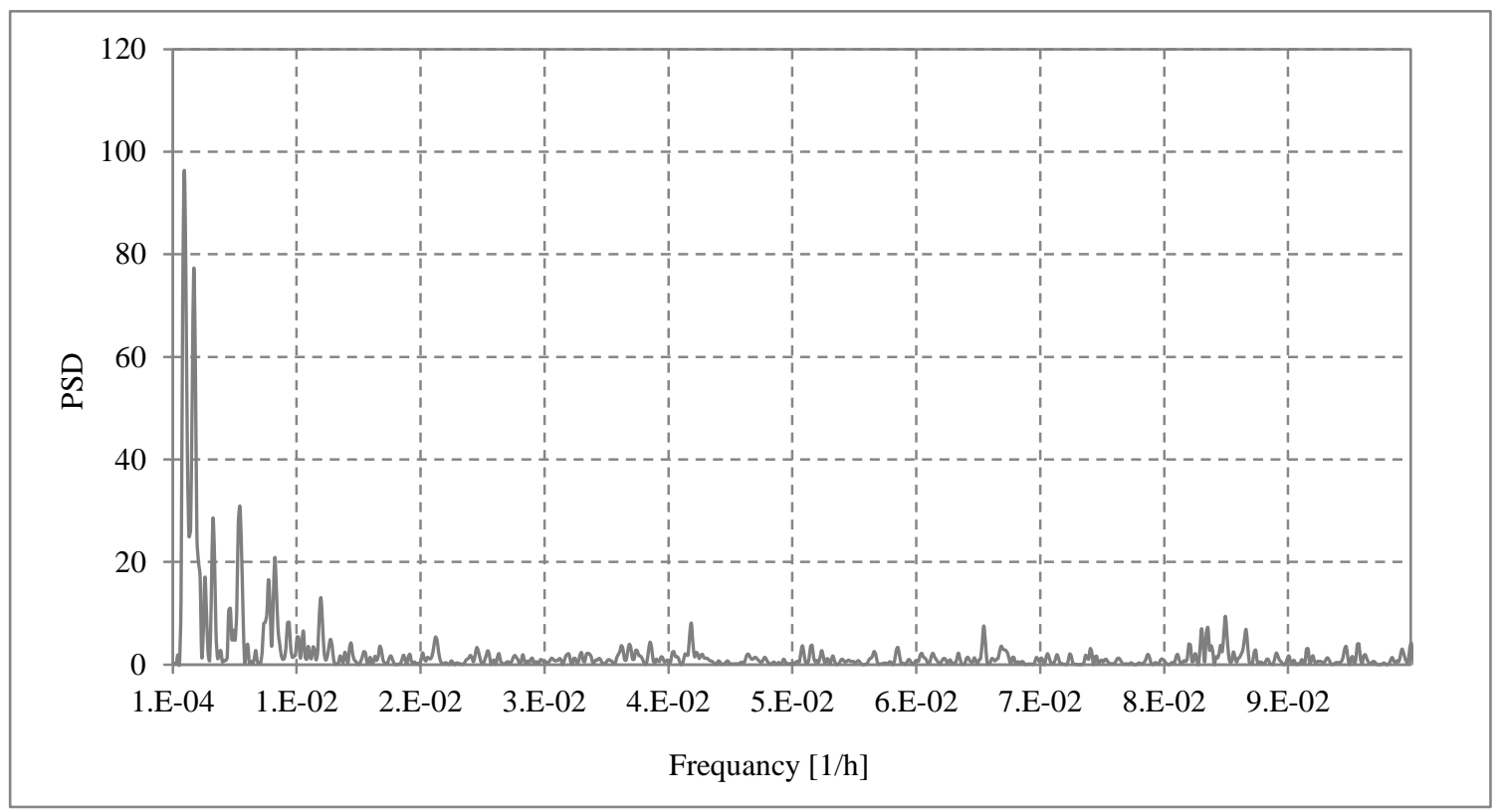

Fig. 5: Power spectral density for the pressure corrected CR recorded by the mini neutron monitor for the considered period of study. The frequency unit is (1/hour).

\section{Conclusion}

A mini-neutron monitor was installed at (KACST) central Saudi Arabia (Riyadh; 24 43; long. 46 40; alt. $613 \mathrm{~m} ; \mathrm{Rc}=14.4 \mathrm{Gv}$ ) to supplement the presently existing CR detectors and to study cosmic ray variations at various time scales. Preliminary results obtained from this detector were presented and discussed. Despite the fact that the installed mini NM has a small size in comparsions with the standard neutron monitors, it presented high abilities to record lowenergy CRs.

\section{Acknowledgments}

We would like to thank King Abdulaziz City for Science and Technology (KACST) for supporting this work. 


\section{References}

[1] P Stoker, 2009. The IGY and beyond: A brief history of ground-based cosmic-ray detectors, Adv. in Space Res. 44.

[2] H. Krüger, et al.,2003. First results of a mobile neutron monitor to inter-calibrate the worldwide community. Proc.28th ICRC.

[3] S.Poluianov, 2015. Mini Neutron Monitors at Concordia Research Station, Central Antarctica. J. Astro. Space Sciences, 32, 4.

[4] B. Heber, et al., 2014. Mini neutron monitor measurements at the Neumayer III station and on the German research vessel Polarstern, Journal of Physics: Conference Series, 632, conference 1; Proc.24th ECRS.

[5] H.Krüger, 2008. A calibration neutron monitor: Energy response and instrumental temperature sensitivity. Journal of Geophysical Research, 113, A8.

[6] L. Dorman, 2004. Cosmic rays in the Earth's atmosphere and underground, Kluwer Academic Publishers, USA.

[7] A. Maghrab, et al., 2014. On the calibration of a single channel cosmic ray particle detector, Proc. SPIE 9154, High Energy, Optical, and Infrared Detectors for Astronomy VI, 91542K

[8] A. Maghrabi, et al., 2012. The KACST muon detector and its application to cosmic-ray variations studies, Adv. in Space Res, 50, 6.

[9] M Mailyan and A. Chilingarian 2010, Investigation of diurnal variations of cosmic ray fluxes measured with using ASEC and NMDB monitors. Adv. in Space Res. 45.

[10] K. Kudela, et al., 2003. On diurnal variation of cosmic rays: statistical study of neutron monitor data including LominskyS^tit. Proc.21st ECRS.

[11] P. Chowdhury, et al., 2010. Evaluation of the short and intermediate term periodicities in cosmic ray intensity during solar cycle 23. Planet Space Sci. 58;7-8.

[12] P. Chowdhury and K. Kudela, 2018. Quasi-periodicities in cosmic rays and time lag with the solar activity at a middle latitude neutron monitor: 1982-2017. Astrophys. Space Sci.,17.

[13] M. El Borie, S. Al Thoyaib, 2002. Power spectrum of cosmic-ray fluctuations during consecutive solar minimum and maximum periods. Solar Phys. 209;2. 